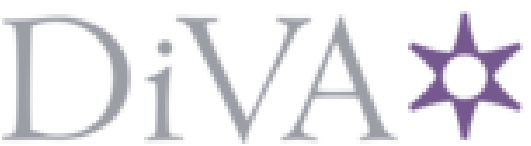

http://www.diva-portal.org

Postprint

This is the accepted version of a paper presented at The International Symposium on Robotics Research (ISRR).

Citation for the original published paper:

Tajvar, P., Varava, A., Kragic, D., Jana, T. (2019)

Robust motion planning for non-holonomicrobots with planar geometric constraints In:

N.B. When citing this work, cite the original published paper.

Permanent link to this version:

http://urn.kb.se/resolve?urn=urn:nbn:se:kth:diva-266371 


\title{
Robust motion planning for non-holonomic robots with planar geometric constraints *
}

\author{
Pouria Tajvar, Anastasiia Varava, Danica Kragic, Jana Tumova \\ KTH Royal Institute of Technology
}

\begin{abstract}
We present a motion planning algorithm for cases where geometry of the robot cannot be neglected and where its dynamics are governed by nonholonomic constraints. While the two problems are classically treated separately, orientation of the robot strongly affects its possible motions both from the obstacle avoidance and from kinodynamic constraints perspective. We adopt an abstraction based approach ensuring asymptotic completeness. To handle the complex dynamics, a data driven approach is presented to construct a library of feedback motion primitives that guarantee a bounded error in following arbitrarily long trajectories. The library is constructed along local abstractions of the dynamics that enables addition of new motion primitives through abstraction refinement. Both the robot and the obstacles are represented as a union of circles, which allows arbitrarily precise approximation of complex geometries. To handle the geometrical constraints, we represent over- and under-approximations of the three-dimensional collision space as a finite set of two-dimensional "slices" corresponding to different intervals of the robot's orientation space. Starting from a coarse slicing, we use the collision space over-approximation to find a valid path and the under-approximation to check for potential path non-existence. If none of the attempts are conclusive, the abstraction is refined. The algorithm is applied for motion planning and control of a rover with slipping without its prior modelling.
\end{abstract}

\section{Introduction}

Various state-of-the-art motion planning approaches for non-holonomic vehicles use simplified models such as the bicycle model to generate feasible trajectories for high level planning [18]. Such models can be either discretized in lattice-based methods or used as a heuristic for measuring distance between two states in sampling-based methods such as probabilistic roadmaps [9]. It is then up to the low-level vehicle feedback controller to follow the prescribed trajectory; an overview of this group of approaches can be found in [15]. This may be a challenge in cases where the simplified model does not resemble the actual vehicle dynamics, which may result in growing error between the prescribed trajectory and vehicles position that in turn may require trajectory re-planning [18]. Such deviations from the prescribed trajectory are particularly challenging when the robot geometry imposes limitations on its possible motion. As an example, consider a robot attempting to exit a narrow passage as illustrated in Fig. 1, where both differential and geometric constraints need to be accounted for.

\footnotetext{
* This work was supported by the EU H2020 Research and Innovation Programme under GA No. 731869 (Co4Robots) and the Knut and Alice Wallenberg Foundation, project IPSYS
} 


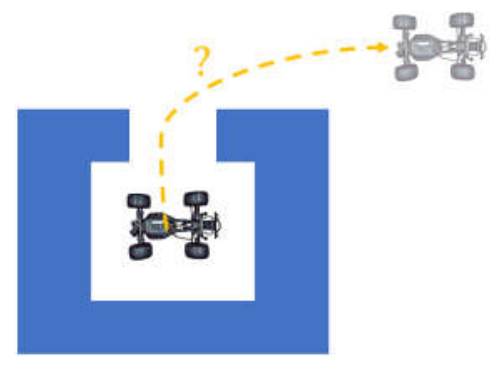

Fig. 1: A motivation scenario: If considered as a purely geometrical problem, the robot controller will not be able to follow the proposed trajectory due to kinodynamic constraints requiring several forward-backward motions to exit. On the other hand, if the robot geometry is not considered, e.g., by considering the robots bounding ball, the problem has no solution.

A class of sampling based motion planning algorithms such as rapidly exploring random trees (RRT) [10] and [7] has been developed to address planning under kinodynamic constraints. These algorithms in contrast to earlier sampling based approaches do not rely on solving a boundary value problem (BVP) for connecting samples in the state space but rather integrate the forward dynamics which makes them suitable for systems with complex dynamics. While asymptotically optimal extensions of RRT such as [8] and [1] again rely on the solution of the BVP problem, [11] has proposed an algorithm that can generate asymptotically near-optimal solutions only using the forward dynamics. The mentioned algorithms are probabilistically complete, i.e. if there exists a solution, the probability of finding a solution approaches 1 as the number of the samples approaches infinity. Path non-existence however, cannot be identified using these methods in contrast with the proposed method in this paper.

Another approach to motion planning is to construct a finite library of feasible motions according to the robot dynamics, i.e. motion primitives, and search for a plan in the discrete motion primitive space $[5,13,17]$. Mobile robots, in contrast to, for example, robotic manipulators, especially benefit from this approach due to the symmetry in their dynamics that allows offline computation of control laws. To address the dynamics uncertainty, methods are proposed recently to use feedback control for motion primitives such that given a bounded disturbance in the dynamics, the state will remain within a bounded margin from the prescribed trajectory [20,13]. However, working with a small set of motion primitives often does not bring the desired results, while a rich set of motion primitives will result in a high branching factor in the discrete space search and increase the computation cost. The completeness property for said algorithms is interchangeably referred to as resolution complete [22] or asymptotically complete [2] i.e. if there exists a solution, a solution will be found given a fine enough resolution in the discretization. In this work we have adopted the term asymptotically complete as the proposed algorithm does not uniformly increase the resolution in case of an undecided result but the refinement of the motion primitive library is guided by the solutions to the sub-problems that are explained in section 2.3. 


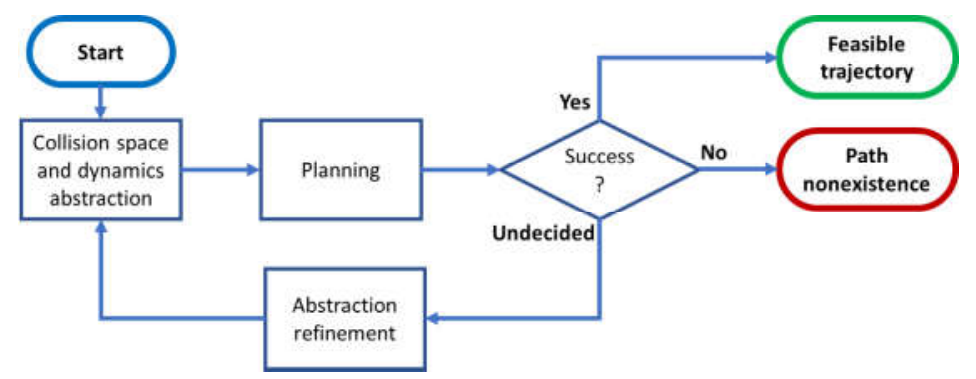

Fig. 2: Approach overview

The problem of verifying path existence based on geometric representation of a robot and an environment has been studied in the context of motion planning [3,21, 14, 22]. It is closely related to the notion of caging from robotic manipulation - a condition under which the mobility of an object is restricted by obstacles or external forces such that it cannot escape arbitrarily far from its initial configuration. In other words, the object is caged if there is no escaping path leading it arbitrarily far from the starting position. Caging and path non-existence verification can be performed by building a discretized representation of the configuration space and analyzing its path-connected components. While the problem of proving path non-existence can be seen as dual to path planning, it is generally more complex, as it implies having information about a path-connectivity of the entire space rather than constructing one particular path. Due to high dimensionality of the state space, constructing its approximation is a computationally expensive procedure $[14,19]$. These approaches however, do not consider kinodynamic constraints on robot motion which can result in infeasible paths for nonholonomic robots.

In this work, we focus on asymptotically complete motion planning with nonholonomic and geometric constraints. We adopt the motion primitive approach and tackle the completeness and branching factor problems through abstraction refinement (see Fig. 2). The abstraction refinement is applied both to the construction of a dynamic library of motion primitives and computation of collision space for an arbitrary robot shape expressed as a union of circles. In order to make state space representation computationally efficient, we represent it as set of "slices", where each slice is a twodimensional representation of the collision space corresponding to a certain interval of orientations. By construction, our approximation provides a proper subset of the real collision space, which means that if our algorithm reports that the two states are disconnected, then there is no path between them in the real free space. As a result, our algorithm is asymptotically complete in both verifying existence of a path and finding a path, if one exists. In our experiment with a rover on a slippery floor we have shown that the overall algorithm is computationally tractable, by constructing a plan to reverse the rover orientation in a tight parking space in 4 seconds. We have further shown that the robust motion primitives are indeed capable of keeping the uncertainty bounded during execution of long trajectories.

The remainder of the paper is organized as follows: In Section 2, the problem is formalized and the overall approach is discussed. In Sections 3, 4, and 5, we introduce 
the details of the three main ingredients of the algorithm: the synthesis of robust motion primitives, the motion planning, and the collision space abstraction through slicing, respectively. Section 6 reports on our experiments with a rover on a slippery floor. Finally, Section 7 concludes the paper and outlines our future research directions.

\section{Problem Definition and Approach}

\subsection{Notation}

Let us denote by $\mathbb{R}$ and $\mathbb{R}_{>0}$ the set of real numbers and positive numbers respectively and by $\mathbb{N}$ the set of natural numbers. An $\varepsilon$-ball of a point $x$ in a space $X$ is denoted by

$$
B_{\mathcal{\varepsilon}}(x)=\{y|| \mid x-y \| \leq \varepsilon\}
$$

and the interior of a set $X$ is defined as

$$
\operatorname{int}(X)=\left\{x \in X \mid \exists \varepsilon>0, B_{\varepsilon}(x) \subset X\right\} .
$$

\subsection{Problem Statement}

Consider an agent whose dynamics is governed by the following discrete-time nonlinear system:

$$
x(t+1)=x(t)+f(x(t), u(t))
$$

with $x(t) \in \mathscr{X} \subseteq \mathbb{R}^{n}$ as system state and $u(t) \in \mathscr{U} \subset \mathbb{R}^{m}$ as system input. Let $\mathscr{X}$ and $\mathscr{U}$ be compact sets.

We consider the case where function $f$ is not explicitly given but can be sampled from. This is a common case for mobile robots with unmodeled dynamics. The underlying continuous-time dynamics of the robot is not considered as the presented datadriven approach is based on time-sampled data.

Let us assume the agent as a rigid body in a $d$-dimensional environment. The configuration space for the agent body is therefore, $\mathbb{R}^{2} \times S O(d)$, where $S O(d)$ corresponds to orientation space for a $d$-dimensional environment. $S O(d)$ is a closed set for all $d \in \mathbb{N}$. Let us assume that orientation space is $s$-dimensional, $S O(d) \subset \mathbb{R}^{s}$. The agent's state can be seen as a composition of three components, i.e.

$$
\mathscr{X}=\mathscr{X}^{c} \times \mathscr{X}^{o} \times \mathscr{X}^{i}
$$

where $\mathscr{X}^{c} \subseteq \mathbb{R}^{d}, \mathscr{X}^{o} \subset S O(d)$, and $\mathscr{X}^{i} \subset \mathbb{R}^{n-d-s}$ correspond to the agent's position in Cartesian space, the agent's orientation, and the agent's internal states (for example velocity), respectively. Let $\Pi_{c o}(x(t))$ be used to refer to the projection of state $x(t)$ into the joint space $\mathscr{X}^{c} \times \mathscr{X}^{o}$. In this paper, we consider objects in planar environment, i.e. $d=2$, and as a result we have $s=1$.

Let us be given an initial state of the agent $x_{0} \in \mathscr{X}$, and a goal region $X_{g} \subset \mathscr{X}$.

The shape of the agent is represented by a union of $n_{\text {agn }} 2$-dimensional balls

$$
\bigcup_{j=1}^{n_{\text {agn }}} B_{r_{j}}\left(p_{j}\right)
$$


corresponding to agent's initial position. Let the obstacles be similarly represented by a union of $n_{o b s} 2$-dimensional balls

$$
\bigcup_{j=1}^{n_{o b s}} B_{r_{j}}\left(p_{j}\right)
$$

The collision space $\mathscr{C} \subset \mathscr{X}^{c} \times \mathscr{X}^{o}$ therefore, corresponds to the set of states with non-empty intersection between the robot and the obstacle.

We define the problem as follows:

Problem 1. Given an agent with translation invariant dynamics (1) and of shape (3), and a set of obstacles (4), either find a sequence of states (i.e. trajectory) $(x(0), x(1), \ldots, x(T))$ and a corresponding sequence of inputs $(u(0), u(1), \ldots, u(T))$ such that:

1. $x(0)=x_{0}$

2. $x(T) \in X_{\text {goal }}$

3. $\forall 0 \leq t \leq T, \Pi_{c o}(x(t)) \notin \mathscr{C}$, i.e. the sequence of state is collision-free, and

4. $\forall 0 \leq t \leq T-1, x(t+1)=x(t)+f(x(t), u(t))$;

or conclude non-existence of such path.

\subsection{Approach}

In this section, we overview our abstraction-based approach to Problem 1. The algorithm modules are depicted in Fig. 3, which refines Fig. 2. We initially start from a coarse discretization of the input space and the orientation space, i.e. the grid and the slices, respectively.

The grid serves as a starting point to build a discrete abstraction of the dynamics. Intuitively, we generate a finite set of motion primitives, one for each grid cell. Each motion primitive is composed of a constant input - the grid cell center - and a feedback control law around each cell center, which ensures that the application of the motion primitive is guaranteed to keep the state uncertainty within a certain bound. The procedure is detailed in Section 3.1. The slices serve to compute an under- and an over-approximation of the collision space, which is described in Section 5.

Having ensured bounded state uncertainty and an under-approximation of the collision space, we attempt to find a sequence of motion primitives satisfying Problem 1. We translate the problem into a planning problem on a discrete graph, where vertices represent centers of $\varepsilon$-balls that do not intersect with the under-approximation of the collision space, and edges are defined by motion primitives driving the system from one center of an $\varepsilon$-ball to another. The feedback control term ensures that regardless of where within the former $\varepsilon$-ball the system is, it will end up within the latter $\varepsilon$-ball. The discrete planning problem can then be addressed e.g., via $A^{*}$.

If a plan is found, we check it against the over-approximation of the collision space. If the plan is collision free then the algorithm uses it to obtain a valid motion plan solving Problem 1. However, there are two possible causes for the plan to intersect with the 


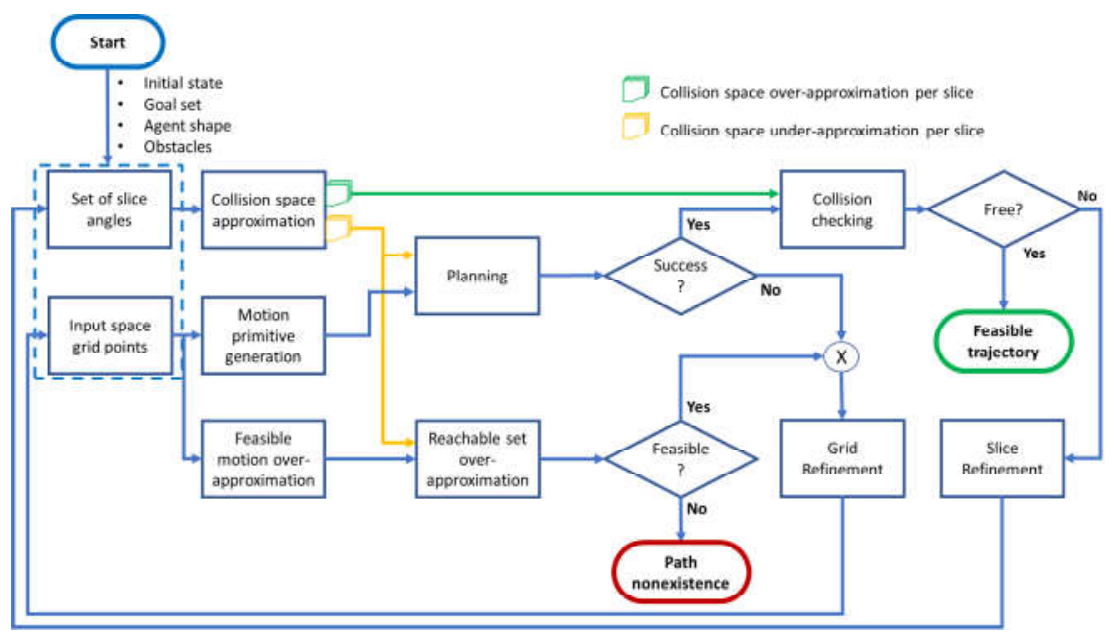

Fig. 3: Approach overview - refined

over-approximation collision space: the first one is the actual non-existence of the desired collision-free trajectory, and the second one is the conservativeness of the collision space over-approximation. To determine the true cause, we refine the discretization of the orientation space collision by splitting each slice in which a collision has occurred and thus decreasing the conservativeness of the over-approximation.

Analogously, there are two different possible reasons for not finding a plan from the initial state to the goal region. We compute an over-approximation of the reachable set to determine, whether the plan does not exist for the original system, or whether the grid was not fine enough to prove or disprove existence of such plan. In the latter case, we refine the grid on the input space, and repeat the procedure.

\section{Input space approximation}

\subsection{Construction of robust motion primitives}

In this section we provide a method to construct motion primitives represented as feedback control laws. Due to linearization error, application of a motion primitive will introduce some uncertainty in the state. The state uncertainty in general grows when applying a sequence of motion primitives. The feedback control presented here is to ensure that the uncertainty will remain within a constant bound for any sequence of motion primitives. Feedback for robotic motion stabilization is presented in [20] for fully actuated systems, i.e. where system state can be controlled in an arbitrary direction, and in [16] for systems where linearization error may be neglected when considering small state neighborhoods. In this section we provide a feedback control synthesis considering a more general class of dynamics encompassing for example standard non-holonomic vehicle models. 
Let us denote the joint state-input space as $Z=\mathscr{X} \times \mathscr{U}$. We write $f$ as a vector of $n$ functions $\left[f_{1}, \ldots, f_{n}\right]^{\top}$, where function $f_{i}: Z \rightarrow \mathbb{R}$ corresponds to the $i$-th output.

Assumption 1 The function $f_{i}$ is twice differentiable and its Hessian is element-wise bounded:

$$
\exists \overline{h_{i}}<\infty \text { s.t. } \max _{j, k \in \mathbb{N}_{<n+m}, z \in Z}\left|\frac{\partial^{2} f_{i}}{\partial z_{j} \partial z_{k}}(z)\right| \leq \overline{h_{i}}
$$

Let us define $f^{1}=f$ and

$$
f^{k+1}\left(x,\left[u_{1}^{\top} \ldots u_{k+1}^{\top}\right]^{\top}\right)=f\left(f^{k}\left(x,\left[u_{1}^{\top} \ldots u_{k}^{\top}\right]^{\top}\right), u_{k+1}\right) .
$$

In other words, $f^{k}$ is a multi-step extension of dynamics $f$. The motivation is that $f$ may not be fully actuated in general in one control step but it may be fully actuated in $k$ control steps. For example a car cannot be controlled to move side-ways with any constant input but can be moved side-ways with a sequence of inputs. Therefore, we eventually construct feedback motion primitives for $k$ step extensions of dynamics to obtain full actuation for stabilization.

Lemma 1. When Assumption 1 holds for a function $f$, it also holds for $f^{k}$ for all $k$.

It is straightforward to show Lemma 1 by applying the chain rule to calculate partial derivatives for $f^{k}$.

Definition 1. $f$ is said to be robustifiable on $\mathscr{X} \times \mathscr{U}$ if and only if for all $x \in \operatorname{int}(\mathscr{X})$ and $u_{1}, \ldots, u_{n} \in \operatorname{int}(\mathscr{U}),\left[\frac{\partial f^{n}}{\partial u_{n}} \ldots \frac{\partial f^{n}}{\partial u_{1}}\right]$ is full rank.

Note that for a linear system $f(x, u)=A x+B u$ robustifiability is equivallent to controllability: We have $\frac{\partial f^{n}}{\partial u_{n}}=B$ and $\frac{\partial f^{n}}{\partial u_{1}}=A^{n-1} B$. In the subsequent sections we assume that $f$ is robustifiable.

Affine approximation Consider a state $x_{0} \in \mathscr{X}$, an input $u_{0} \in \mathscr{U}$ and two arbitrary vectors $\sigma_{x} \in \mathbb{R}_{>0}^{n}$ and $\sigma_{u} \in \mathbb{R}_{>0}^{m} . \sigma_{x}$ and $\sigma_{u}$ correspond to the diagonals of boxes in the state space and the input space respectively.

We define a region in the state-input space

$$
X \times U=\left\{x_{0}\right\} \oplus\left[-\sigma_{x}, \sigma_{x}\right] \times\left\{u_{0}\right\} \oplus\left[-\sigma_{u}, \sigma_{u}\right],
$$

where the operator $\oplus$ is used to denote Minkowski sum of two sets: $X \oplus Y=\{x+y \mid x \in$ $X, y \in Y\}$.

Definition 2. $\bar{f}(x, u)=\{A x+B u+c\} \oplus \mathbf{W}$ is a conservative affine approximation of $f$ in $X \times U$ if:

$$
\forall x \in X, u \in U f(x, u) \in \bar{f}(x, u) .
$$

As shown in [4], such approximation can be constructed with finite samples for a function under Assumption 1 and the linearization error bound for $i$-th output, i.e. $\left|W_{i}\right|$ can be calculated as follows:

$$
\left|W_{i}\right|=\bar{h}_{i} \sum_{j=1}^{n+m} \sum_{k=1}^{n+m} \sigma_{z_{j}} \sigma_{z_{k}}
$$


Error bound invariance We show that under the assumptions stated for $f$, i.e. the bounded Hessian and robustifiability, it is possible to find a bounding box size $\sigma_{x}$ such that, if the state uncertainty is within $\sigma_{x}$ at time step $t$, it can be kept within the $\sigma_{x}$ at step $t+n$ through state feedback.

Theorem 1. For a robustifiable system $x^{+}=f(x, u)$, for any $\tilde{x} \in \operatorname{int}(\mathscr{X})$ and $\tilde{u}_{1}, \ldots, \tilde{u}_{n} \in$ $\operatorname{int}(\mathscr{U})$ there exists $\sigma_{x} \in \mathbb{R}_{>0}^{n}, \sigma_{u} \in \mathbb{R}_{>0}^{m}$, and a control law $C: \mathbb{R}^{n} \rightarrow\left(\mathbb{R}^{m}\right)^{n}$ such that $\forall x \in\{\tilde{x}\} \oplus\left[-\sigma_{x}, \sigma_{x}\right]:$

1. $C_{t}(x) \in \tilde{u}_{t} \oplus\left[-\sigma_{u}, \sigma_{u}\right] \subseteq \mathscr{U}$.

2. $f^{n}(x, C(x)) \in f^{n}(\tilde{x}, \tilde{u}) \oplus\left[-\sigma_{x}, \sigma_{x}\right] \subseteq \mathscr{X}$.

With $\tilde{u}=\left[\tilde{u}_{1}^{\top}, \ldots, \tilde{u}_{n}^{\top}\right]^{\top}$ and $C$ being a mapping between from state to the sequence of inputs for $n$ time steps of $f^{n}$ and $C_{t}$ corresponding to $t$-th time step.

Proof. We have from Lemma 1 that Assumption 1 holds for $f^{n}$. Therefore, we can conservatively approximate $f^{n}$ for an arbitrary $X=\{\tilde{x}\} \oplus\left[-\sigma_{x}, \sigma_{x}\right]$ and $U=\tilde{u}_{t} \oplus\left[-\sigma_{u}, \sigma_{u}\right]$. Choice of $\sigma_{x}$ is only subject to the constraint $X \in \mathscr{X}$ but is guaranteed to be non-empty as $\tilde{x} \in \operatorname{int}(\mathscr{X})$, and similarly for $\sigma_{u}$. With a slight abuse of notation, we will use $u$ to denote the total sequence of inputs in the proof. We will have:

$$
\forall x \in X, u \in U f^{n}(x, u) \in \overline{f^{n}}(x, u) .
$$

with $\overline{f^{n}}(x, u)=A x+B u+c+\mathbf{W}$. Since the approximation matches the nonlinear function in the linearization point by construction we can write $\overline{f^{n}}(x, u)=A(x-\tilde{x})+B(u-$ $\tilde{u})+f^{n}(\tilde{x}, \tilde{u})+\mathbf{W}$

By a coordination shift to $(\tilde{x}, \tilde{u})$ and subtraction of constant term $f^{n}(\tilde{x}, \tilde{u})$ we write $\overline{f^{n}}(x, u)=A x+B u+\mathbf{W}$.

As the system is robustifiable, we have that $B$ is full rank and as a result is pseudoinvertible. Consider the control rule $u=C(x)=-B^{\#} A x$, with $B^{\#}$ being the pseudoinverse of $B$. We calculate the value $\varepsilon^{*}$ as: $\varepsilon^{*}=\max _{\mathcal{E}}$ s.t. $B_{\varepsilon}(0) \subset\left[-\sigma_{u}, \sigma_{u}\right]$.

From $\sigma_{u}>0$ we get $\varepsilon^{*}>0$. We now calculate a value $\alpha$ as follows:

$$
\alpha=\max _{x \in\left[-\sigma_{x}, \sigma_{x}\right]} \frac{\left\|-B^{\#} A x\right\|_{\infty}}{\varepsilon^{*}} .
$$

If $\alpha<1$, the values of the input will remain inside the enclosed circle within the feasible inputs and therefore, satisfy first condition in Theorem 1 . If $\alpha>1$ we can scale the choice of $\sigma_{x}$ by a value smaller than $\frac{1}{\alpha}$, as the controller is linear with the state, the input will be bounded inside $\varepsilon^{*}$.

Now with the control $u=-C(x)=-B^{\#} A x x$ we get $\overline{f^{n}}\left(x,-B^{\#} A x\right)=\mathbf{W}$. Therefore, for the second condition in Theorem 1 to hold we need: $\mathbf{W} \subseteq\left[-\sigma_{x}, \sigma_{x}\right]$ or equivalently:

$$
\beta=\max _{i \in \mathbb{N}_{\leq n}} \frac{\left|W_{i}\right|}{\sigma_{x_{i}}} \leq 1 .
$$

If $\beta>1$, the second condition is violated. We can observe from (6) that if we scale $\sigma_{z}=\left[\sigma_{x}^{\top} \sigma_{u}^{\top}\right]^{\top}$ by $\frac{1}{\beta},\left|W_{i}\right|$ will be scaled by $\frac{1}{\beta^{2}}$ and therefore, the second condition becomes satisfied. We note that mutual scaling of $\sigma_{x}$ and $\sigma_{u}$ does not affect the first condition. Therefore, by starting from an arbitrary $\sigma_{x}$ and $\sigma_{u}$, we can calculate $\sigma_{x}^{\prime}=\frac{\sigma_{x}}{\alpha \beta}$ and $\sigma_{u}^{\prime}=\frac{\sigma_{u}}{\beta}$ that satisfy both conditions from Theorem 1 . 
Reachable set in 1 time step Reachable set in 2 time steps Reachable set in $\mathbf{3}$ time steps
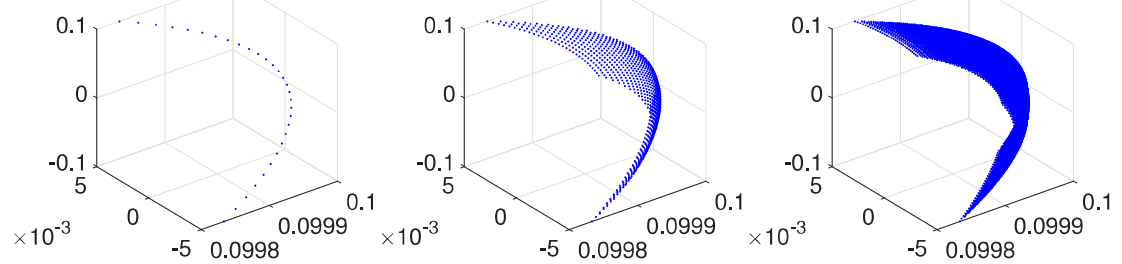

Fig. 4: Reachable set for fixed velocity Reeds-Shepp model with different time steps controlled with steering angle - In 1 and 2 time steps the reachable sets are degenerate, i.e. 1 and 2 dimensional manifolds respectively, therefore, the state error cannot be fully compensated through feedback. In 3 time steps however, the reachable set is a volume so the state error in any direction can be compensated.

Following similar steps we can show that the conditions hold for $\sigma_{x}^{\prime \prime}=\frac{\gamma \sigma_{x}}{\alpha \beta}$ and $\sigma_{u}^{\prime \prime}=\frac{\gamma \sigma_{u}}{\beta}$ for all $0<\gamma \leq 1$, which means that we can have motion primitives with arbitrarily low invariant state error for a deterministic robustifiable nonlinear system under Assumption 1.

Example: Reeds-Shepp car model In this example, we discuss that the common Reeds-Shepp car model satisfies the assumptions required for construction of robust motion primitives. Consider the continuous time Reeds-Shepp car model:

$$
\left[\begin{array}{l}
x \\
y \\
\theta
\end{array}\right]=\left[\begin{array}{c}
u_{1} \cos (\theta) \\
u_{1} \sin (\theta) \\
u_{1} u_{2}
\end{array}\right]
$$

As can be seen in Fig. 4, the 3-step reachable set by controlling the steering angle is non-degenerate, i.e. full dimensional, therefore, we expect $f^{3}$ to be robustifiable. To verify, we can compute the exact time-discretization of (8) for time period $\tau$ as follows:

$$
f\left(\left[\begin{array}{l}
x \\
y \\
\theta
\end{array}\right]\right)=\left[\begin{array}{l}
x \\
y \\
\theta
\end{array}\right]+\left\{\begin{array}{cc}
{\left[\begin{array}{c}
u_{1} \cos (\theta) \tau \\
u_{1} \sin (\theta) \tau \\
0
\end{array}\right]} & \text { for } u_{2}=0 \\
{\left[\begin{array}{c}
\frac{1}{u_{2}}\left(\sin \left(\theta+u_{1} u_{2} \tau\right)-\sin (\theta)\right) \\
\frac{1}{u_{2}}\left(\cos \left(\theta+u_{1} u_{2} \tau\right)-\cos (\theta)\right) \\
u_{1} u_{2} \tau
\end{array}\right]} & \text { for } u_{2} \neq 0
\end{array}\right.
$$

Note that although in case of Reeds-Shepp model we can compute explicit timediscretization, this is not required in general in our approach as motion primitives are constructed using samples from the dynamics and rely only on the Lipschitz continuity assumption.

Without loss of generality due to the continuous model symmetry, we can assume state to be $[0,0,0]^{\top}$. We compute the 3 -step controllability matrix for $f^{3}$ when 1 - con- 

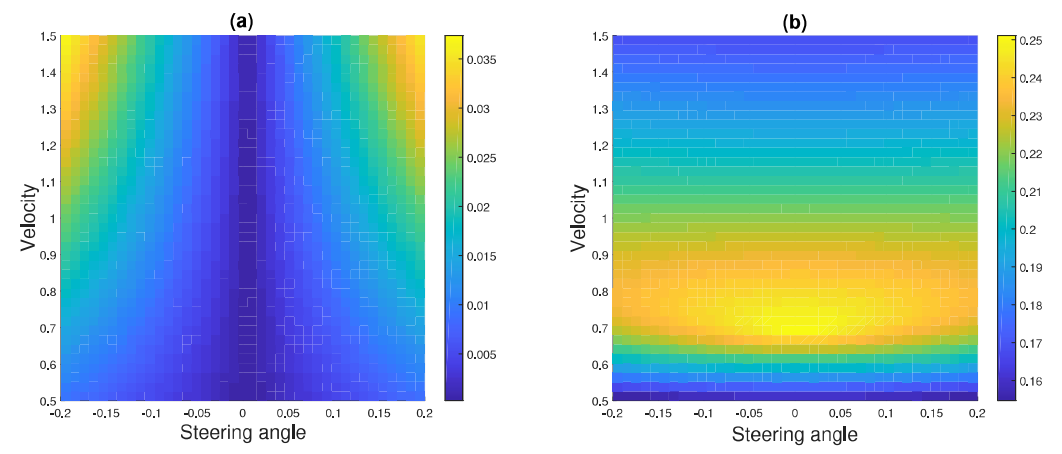

Fig. 5: Measuring robustifiablity of Reeds-Shepp model when controlled (a) through feedback on steering angle only and (b) through feedback on steering angle and velocity.

trolled through feedback on steering angle only (keeping velocity constant) and 2- feedback on steering angle along with velocity; the controllability matrices are $3 \times 3$ and $3 \times 6$ respectively. One way to verify whether the system is robustifiable around an input is through singular value decomposition. Singular values, correspond to magnitude of displacement in orthogonal directions in the state space given fixed magnitude of displacement in the input space. In case of degenerate controllable matrices, i.e. nonrobustifiable motions, one or more singular values will be equal to zero meaning that the state cannot be pushed toward certain directions. In Fig. 5. the ratio between the smallest singular value and the largest singular value, i.e. inverse of matrix condition number, can demonstrate a measure of robustifiability. As seen in Fig. 5. while the model is robustifiable for almost entire input space when controlled only with the steering angle, the matrix becomes ill conditioned close to zero steering angle, it can also be seen in Fig. 4. that although the reachable set is not degenerate in 3 time steps, the volume is rather thin in one direction. However, when controlled with both steering angle and velocity, the model is robustifiable and reasonably well conditioned in entire input space with smallest to largest singular value ratio remaining above 0.15 . Feedback on velocity alone is not demonstrated as the solution remains degenerate regardless of time steps since given a fixed steering angle the car will only be able to move on a circle or a straight line.

\subsection{Over-approximation of the reachable set}

We showed that feedback controller can be designed around a robustifiable state-input point such that the state uncertainty does not grow through execution of motion primitives. As the controller is restricted to a discrete set of motion primitives, it does not capture all possible motions of the robot. Therefore, in this section we present a method to over-approximate all possible motions, i.e. reachable set, of the robot.

As shown in Fig. 6 we define a neighborhood around the central input for each motion primitive such that we cover the entire input space with the neighborhoods. For each neighborhood the system dynamics can be linearized around the central input. The 

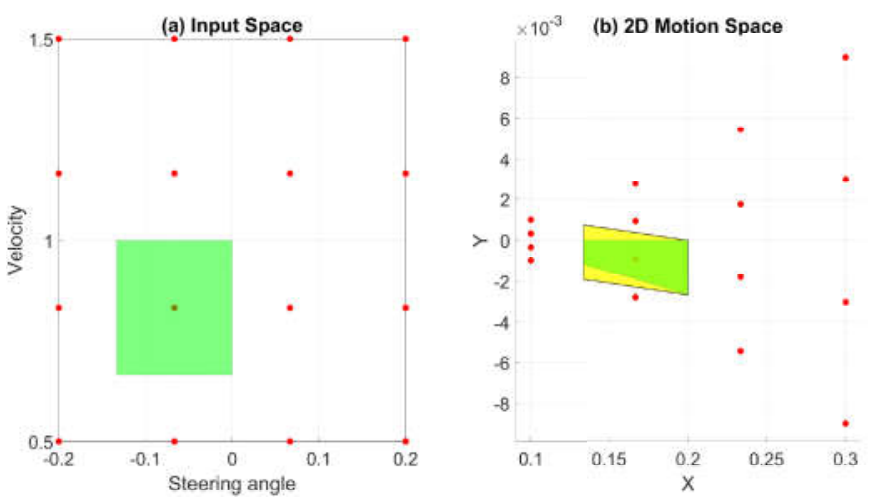

Fig. 6: Reachable set over-approximation: Red dots in (a) show samples from the input space representing central inputs for motion primitives. Red dots in (b) are corresponding motions projected to 2D Cartesian space. The green box in (a) shows the neighborhood of one of the motion primitives and the green and the yellow patches in (b) show corresponding reachable set and its over-approximation respectively.

over-approximation of the reachable set can be computed as the Minkowski sum of the linear transformation of the neighborhood and the linearization error in the neighborhood that can be derived from the Lipschitz constant of the dynamics. In practical cases where the Lipschitz constant is unknown, the linearization error can be computed as an optimization problem on samples from the neighborhood.

\section{Motion planning}

With construction of the robust motion primitives, we now have a discrete action space that due to the uncertainty preserving property, can be chained to synthesize an arbitrarily long sequence, i.e. trajectory. We translate the problem into a planning problem on a discrete graph, where vertices represent centers of $\varepsilon$-balls that do not intersect with the under-approximation of the collision space, and edges are defined by motion primitives driving the system from one center of an $\varepsilon$-ball to another. The feedback control term ensures that regardless of where within the former $\varepsilon$-ball the system is, it will end up within the latter $\varepsilon$-ball. One of the common approaches of planning with a discrete set of actions is the $A^{*}$ which is a graph search algorithm that is guided with a heuristic that under-approximates the distance from each state to the goal. For mobile robotics applications, various extensions of $\mathrm{A}^{*}$ have been proposed to allow differential constraints and increase computation speed by allowing sub-optimal solutions [6]. In this work we have used the anytime repairing $\mathrm{A}^{*}$ (ARA*) for planning [12]. In $\mathrm{ARA}^{*}$, the graph search is initiated with an inflated heuristic (with a multiplier $\Gamma>1$ ) and gradually reduces $\Gamma$ to 1 . ARA* can provide a provably optimal solution if run sufficiently long but in general provides sub-optimal solutions with shrinking bounds on sub-optimality. It is shown to be efficient for finding solutions in graph search with high branching factors. 


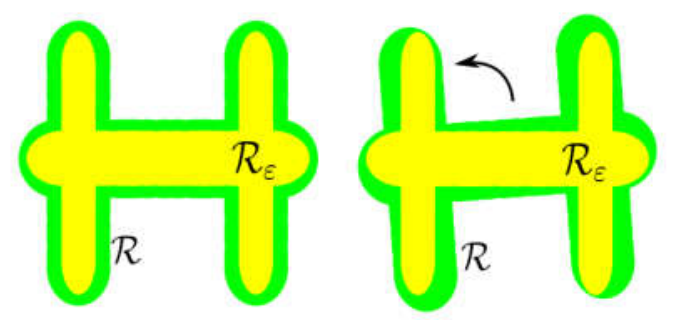

Fig. 7: The robot is depicted in green, and its $\varepsilon$-core is depicted in yellow. Left: both the robot and its $\varepsilon$-core have the same orientation. Right: the robot is rotated such that the $\varepsilon$-core stays inside despite its orientation is fixed.

\section{Collision space approximation}

In this section, we introduce our algorithm for constructing over- and under- approximations of the collision space of the object for fixed orientation intervals.

Let us start with under-approximation construction. For a given orientation interval $\left[\theta_{1}, \theta_{2}\right]$, we take it's middle $\theta_{m}=\frac{\theta_{1}+\theta_{2}}{2}$ and compute the corresponding projection $\pi_{\mathbb{R}^{2}}^{\theta=\theta_{m}}\left(\mathscr{C}^{\text {col }}\left(\mathscr{R}_{\varepsilon_{\text {core }}}\right)\right)$ of the collision space onto $\mathbb{R}^{2}$. To see how this projection is related to any other projection $\pi_{\mathbb{R}^{2}}^{\theta=\theta^{\prime}}\left(\mathscr{C}^{c o l}\left(\mathscr{R}_{\varepsilon_{\text {core }}}\right)\right)$, where $\theta^{\prime}$ is another fixed orientation from the same interval $\left[\theta_{1}, \theta_{2}\right]$, we use the notion of $\varepsilon$-core introduced in [19], see Fig. 7.

Definition 3. Let us denote the shape of the robot by $\mathscr{R}$. An $\mathcal{E}$-core of the robot, denoted by $\mathscr{R}_{\varepsilon}$, is the subset of $\mathscr{R}$ consisting of the points of $\mathscr{R}$ that are located at a distance $\varepsilon$ or higher from the boundary of $\mathscr{R}$ :

$$
\mathscr{R}_{\varepsilon}=\{x \in \mathscr{R}: d(\partial \mathscr{R}, x) \geq \varepsilon\}
$$

Imagine an $\varepsilon$-core $\mathscr{R}_{\varepsilon}$ placed inside of the robot $\mathscr{R}$. If we fix the orientation of $\mathscr{R}$ and rotate $\mathscr{R}_{\varepsilon}$ around the center of mass, there is an orientation interval for which $\mathscr{R}_{\varepsilon}$ stays strictly inside $\mathscr{R}$, see Fig. 7. The size of this interval can be determined based on the value $\varepsilon$ according to the following inequality [19]:

$$
2|\sin (\phi / 2)| \cdot \max _{y \in \mathscr{R}}\|y\|,
$$

where $\phi$ is the rotation angle, and the origin coincides with the center of mass of the robot.

If we now consider any other orientation $\theta^{\prime}$ from the interval $\left[\theta_{1}, \theta_{2}\right]$, the corresponding projection $\pi_{\mathbb{R}^{2}}^{\theta=\theta^{\prime}}\left(\mathscr{C}^{c o l}\right)\left(\mathscr{R}_{\varepsilon}\right)$ is contained strictly inside $\pi_{\mathbb{R}^{2}}^{\theta=\theta_{m}}\left(\mathscr{C}^{c o l}\left(\mathscr{R}_{\varepsilon_{\text {core }}}\right)\right)$, and therefore, we obtain an under-approximation of the collision space.

To obtain its over-approximation, we adopt a similar strategy. Here, instead of considering an $\varepsilon$-core, which is a subset of the robot $\mathscr{R}$, we instead consider its superset an inflated version of the robot. We call it $\delta$-superset and define it as follows: 


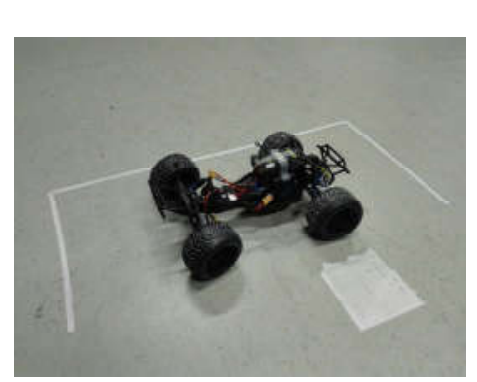

(a)

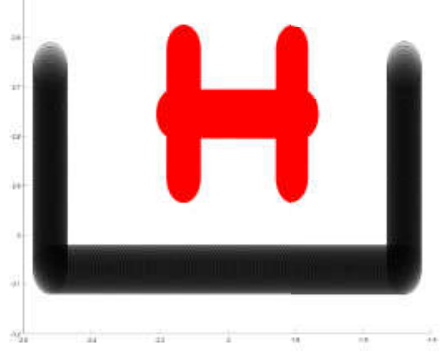

(b)

Fig. 8: Main scenario with the rover reverting its orientation in a small parking area. Left: Erle Rover used on slippery floor. Right: Representation of the rover geometry and the obstacles as a union of circles.

Definition 4. A $\delta$-superset of the robot $\mathscr{R}$, denoted by $\mathscr{R}^{\delta}$, is the superset of $\mathscr{R}$, consisting of $\mathscr{R}$ and the points that are located at a distance $\delta$ or lower from the boundary of $\mathscr{R}$ :

$$
\mathscr{R}^{\delta}=\mathscr{R} \cup\left\{x \in \mathbb{R}^{2}-\mathscr{R}: d(\partial \mathscr{R}, x) \leq \delta\right\}
$$

Similar to the case of under-approximation, we consider a fixed orientation $\theta_{m}=$ $\left(\theta_{1}+\theta_{2}\right) / 2$ and compute the over-approximation of the collision space as a projection of the collision space of its $\delta$-superset: $\pi_{\mathbb{R}^{2}}^{\theta=\theta^{\prime}}\left(\mathscr{C}^{\text {col }}\left(\mathscr{R}^{\delta}\right)\right)$. As in the previous case, as long as the value of $\delta$ is larger than any possible displacement of any point of $\mathscr{R}$ induced by a rotation between different orientations from $\left[\theta_{1}, \theta_{2}\right]$, the robot $\mathscr{R}$ is guaranteed to be strictly inside $\mathscr{R}^{\delta}$, and therefore, we obtain an over-approximation of the collision space.

\section{Experiments}

We tested our approach on an Erle-Rover Unmanned Ground Vehicle (UGV) in a room with motion capture for positioning and slippery floor. The main scenario is to have the rover reverse its heading in a tight parking space, see Fig. 8. The non-holonomy, dynamics uncertainty and the geometrical collision checking are the main challenges. The rover shape is represented as a union of 100 circles and the collision space is represented as a union of 300 circles, see Fig. 8. The algorithm is run on MATLAB communicating with the rover through ROS. Collision space computation is in $\mathrm{C}++$ called during run-time from MATLAB.

We start the input space discretization with 6 motion primitives, corresponding to going left, straight and right in forward and backward drive. To construct the robust motion primitives, we took $5 \times 5$ samples in the corresponding cell to each motion primitive and computed the linearized model and the disturbance bound through a linear program 

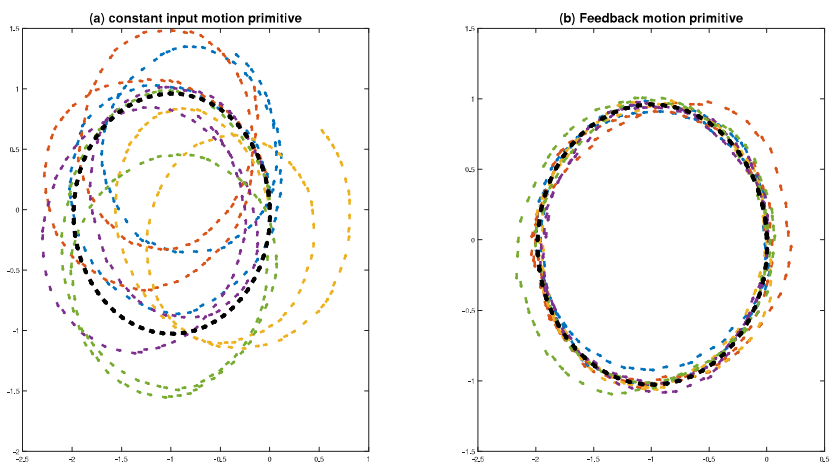

Fig. 9: Implementation of a left turning motion primitive on slippery floor: a) without feedback and b) with the proposed feedback motion primitives. Black arrows mark the reference trajectory and the colored arrows are different runs.

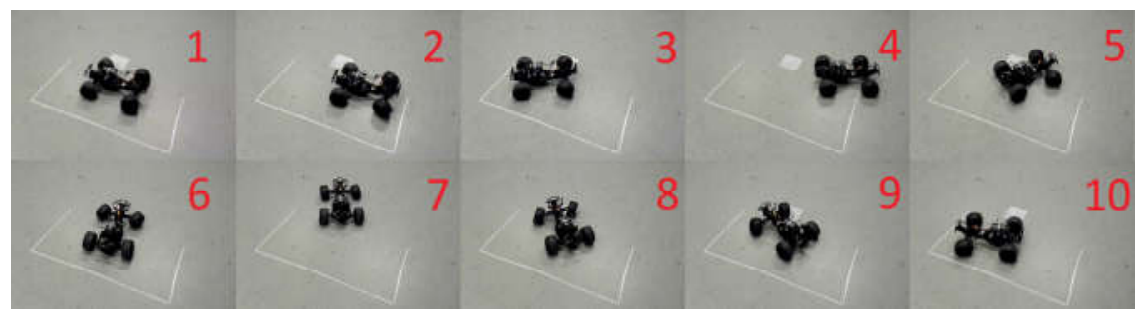

Fig. 10: Rover reversing in the tight parking space.

optimizing for minimum $H_{1}$ norm. We then design uncertainty preserving controllers for all the possible 3 -step combinations of the motion primitives, i.e. $6 \times 6 \times 6$ following the procedure in Section 3.1.

To verify the effectiveness of the feedback motion primitives in maintaining bounded error in presence of dynamics uncertainty, we have compared the rover trajectories when applying only the constant motion primitive and when applying corresponding feedback rule. The result is shown for the left turning motion primitive in Fig. 9.

We initiate the collision space approximation with 100 slices. A discrete plan for the under-approximation of the collision space is generated in 4 seconds, visiting about 1200 nodes and generating a path of length 23 . The successful plan is generated using initial 6 motion primitives without input space refinement. Given the level of dynamics uncertainty, the robot motion could not benefit from a finer sampling in the input space than the 3-by-2 initial grid.

Different stages of the robot trajectory are shown in Fig. 10. We can see that the robot successfully reverts its position in the small parking area without colliding with the defined obstacles. 


\section{Conclusion}

In this paper we presented a motion planning algorithm for systems with non-holonomic dynamics and non-trivial geometry based on abstraction refinement. The abstraction of the collision space converges to the actual collision space for infinitesimal slices and the motion primitive library converges to the entire input space for infinitesimal input grid cells in deterministic systems. Therefore, the proposed algorithm is asymptotically complete for deterministic systems both for finding a plan in case of existence and for verifying path non-existence.

The size of the graph treated by discrete planner grows exponentially with the number of input grid refinements. However, for various practical applications, similar to the rover example used here, the model may not require high input space refinement, because of uncertainty in system dynamics and potential disturbance. Similarly, the worst-case complexity of collision space refinement is exponential, however, only the slices corresponding to uncertain collisions in a planned trajectory are refined.

In the future, we will explore methods of state and input space division besides grid based division to improve scalability to systems with higher space dimensions. We also aim to extend the uncertainty preserving motions to general state-space samples and approaches for guiding state-space sampling through the slice representation of the collision space.

\section{References}

1. Oktay Arslan and Panagiotis Tsiotras. Use of relaxation methods in sampling-based algorithms for optimal motion planning. In 2013 IEEE International Conference on Robotics and Automation, pages 2421-2428. IEEE, 2013.

2. Jérôme Barraquand and Jean-Claude Latombe. Nonholonomic multibody mobile robots: Controllability and motion planning in the presence of obstacles. Algorithmica, 10(2-4):121, 1993.

3. Julien Basch, Leonidas J Guibas, David Hsu, and An Thai Nguyen. Disconnection proofs for motion planning. In Proceedings 2001 ICRA. IEEE International Conference on Robotics and Automation (Cat. No. 01CH37164), volume 2, pages 1765-1772. IEEE, 2001.

4. Thao Dang, Oded Maler, and Romain Testylier. Accurate hybridization of nonlinear systems. In Proceedings of the 13th ACM international conference on Hybrid systems: computation and control, pages 11-20. ACM, 2010.

5. Emilio Frazzoli, Munther A Dahleh, and Eric Feron. Maneuver-based motion planning for nonlinear systems with symmetries. IEEE transactions on robotics, 21(6):1077-1091, 2005.

6. David González, Joshué Pérez, Vicente Milanés, and Fawzi Nashashibi. A review of motion planning techniques for automated vehicles. IEEE Transactions on Intelligent Transportation Systems, 17(4):1135-1145, 2016.

7. David Hsu, Robert Kindel, Jean-Claude Latombe, and Stephen Rock. Randomized kinodynamic motion planning with moving obstacles. The International Journal of Robotics Research, 21(3):233-255, 2002.

8. Sertac Karaman and Emilio Frazzoli. Sampling-based algorithms for optimal motion planning. The international journal of robotics research, 30(7):846-894, 2011.

9. Lydia E Kavraki, Petr Svestka, J-C Latombe, and Mark H Overmars. Probabilistic roadmaps for path planning in high-dimensional configuration spaces. IEEE transactions on Robotics and Automation, 12(4):566-580, 1996. 
10. Steven M LaValle. Rapidly-exploring random trees: A new tool for path planning. 1998.

11. Yanbo Li, Zakary Littlefield, and Kostas E Bekris. Asymptotically optimal sampling-based kinodynamic planning. The International Journal of Robotics Research, 35(5):528-564, 2016.

12. Maxim Likhachev, Geoffrey J Gordon, and Sebastian Thrun. Ara*: Anytime $\mathrm{a}^{*}$ with provable bounds on sub-optimality. In Advances in neural information processing systems, pages 767-774, 2004.

13. Anirudha Majumdar and Russ Tedrake. Funnel libraries for real-time robust feedback motion planning. The International Journal of Robotics Research, 36(8):947-982, 2017.

14. Zoe McCarthy, Timothy Bretl, and Seth Hutchinson. Proving path non-existence using sampling and alpha shapes. In 2012 IEEE International Conference on Robotics and Automation, pages 2563-2569. IEEE, 2012.

15. Brian Paden, Michal Č́p, Sze Zheng Yong, Dmitry Yershov, and Emilio Frazzoli. A survey of motion planning and control techniques for self-driving urban vehicles. IEEE Transactions on intelligent vehicles, 1(1):33-55, 2016.

16. Alejandro Perez, Robert Platt, George Konidaris, Leslie Kaelbling, and Tomas LozanoPerez. Lqr-rrt*: Optimal sampling-based motion planning with automatically derived extension heuristics. In 2012 IEEE International Conference on Robotics and Automation, pages 2537-2542. IEEE, 2012.

17. Janko Petereit, Thomas Emter, Christian W Frey, Thomas Kopfstedt, and Andreas Beutel. Application of hybrid a* to an autonomous mobile robot for path planning in unstructured outdoor environments. In ROBOTIK 2012; 7th German Conference on Robotics, pages 1-6. VDE, 2012

18. Philip Polack, Florent Altché, Brigitte d'Andréa Novel, and Arnaud de La Fortelle. The kinematic bicycle model: A consistent model for planning feasible trajectories for autonomous vehicles? In 2017 IEEE Intelligent Vehicles Symposium (IV), pages 812-818. IEEE, 2017.

19. Anastasiia Varava, J Frederico Carvalho, Florian T Pokorny, and Danica Kragic. Free space of rigid objects: Caging, path non-existence, and narrow passage detection. In Workshop on Algorithmic Foundations of Robotics, 2018.

20. Marijan Vukosavljev, Zachary Kroeze, Mireille E Broucke, and Angela P Schoellig. A framework for multi-vehicle navigation using feedback-based motion primitives. In 2017 IEEE/RSJ International Conference on Intelligent Robots and Systems (IROS), pages 223229. IEEE, 2017

21. Liangjun Zhang, Young J Kim, and Dinesh Manocha. A simple path non-existence algorithm using c-obstacle query. In Algorithmic Foundation of Robotics VII, pages 269-284. Springer, 2008.

22. Bo Zhou, Yi-Jen Chiang, and Chee Yap. Soft subdivision motion planning for complex planar robots. In 26th Annual European Symposium on Algorithms (ESA 2018). Schloss Dagstuhl-Leibniz-Zentrum fuer Informatik, 2018. 